\title{
Engineering the Structure and Properties of DNA-Nanoparticle Superstructures Using Polyvalent Counterions
}

\author{
Leo Y. T. Chou, Fayi Song, and Warren C. W. Chan
}

Version Post-Print/Accepted Manuscript

Citation L. Chou, F. Song, W. C. W. Chan, "Engineering the Structure and (published version) Properties of DNA-Nanoparticle Superstructures Using Polyvalent Counterions," Journal of the American Chemical Society, 2016, 138 (13), pp 4565-4572.

Publisher's Statement This document is the Accepted Manuscript version of a Published Work that appeared in final form in Journal of the American Chemical Society, copyright (CAmerican Chemical Society after peer review and technical editing by the publisher. To access the final edited and published work see http://dx.doi.org/10.1021/jacs.6b00751.

\section{How to cite TSpace items}

Always cite the published version, so the author(s) will receive recognition through services that track citation counts, e.g. Scopus. If you need to cite the page number of the TSpace version (original manuscript or accepted manuscript) because you cannot access the published version, then cite the TSpace version in addition to the published version using the permanent URI (handle) found on the record page. 


\title{
Engineering the Structure and Properties of DNA-Nanoparticle Superstructures Using Polyvalent Counterions
}

\author{
Leo Y. T. Chou, ${ }^{\dagger, \nabla, \#}$ Fayi Song, ${ }^{\dagger, \nabla}$ and Warren C. W. Chan ${ }^{* \dagger, \dagger, \S, \|, \perp}$
}

${ }^{\dagger}$ Institute of Biomaterials and Biomedical Engineering, Rosebrugh Building, Room 407, 164 College Street, Toronto, Ontario M5S 3G9, Canada

${ }^{\ddagger}$ Terrence Donnelly Centre for Cellular and Biomolecular Research, University of Toronto, 160 College Street, Room 230, Toronto, Ontario M5S 3E1, Canada

${ }^{\S}$ Department of Chemical Engineering, University of Toronto, 200 College Street, Toronto, Ontario M5S 3E5, Canada

"Department of Chemistry, University of Toronto, 80 St George Street, Toronto, Ontario M5S 3H6, Canada

${ }^{\perp}$ Department of Material Science and Engineering, University of Toronto, Wallberg Building, 184 College Street, Suite 140, Toronto, Ontario M5S 3E4, Canada

Supporting Information

ABSTRACT: DNA assembly of nanoparticles is a powerful approach to control their properties and prototype new materials. However, the structure and properties of DNA-assembled nanoparticles are labile and sensitive to interactions with counterions, which vary with processing and application environment. Here we show that substituting polyamines in place of elemental counterions significantly enhanced the structural rigidity and plasmonic properties of DNA-assembled metal nanoparticles. These effects arose from the ability of polyamines to condense DNA and cross-link DNA-coated nanoparticles. We further used polyamine wrapped DNA nanostructures as structural templates to seed the growth of polymer multilayers via layer-bylayer assembly, and controlled the degree of DNA condensation, plasmon coupling efficiency, and material responsiveness to environmental stimuli by varying polyelectrolyte composition. These results highlight counterion

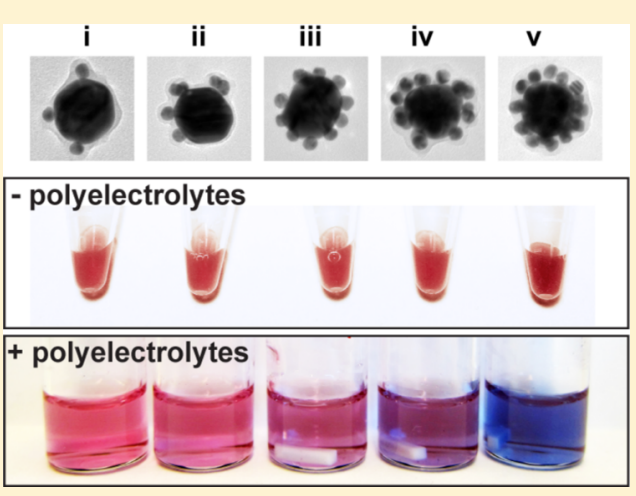
engineering as a versatile strategy to tailor the properties of DNA-nanoparticle assemblies for various applications, and should be applicable to other classes of DNA nanostructures.

\section{INTRODUCTION}

Molecular assembly using DNA benefits from its well-defined structure, sequence programmability, and robust WatsonCrick pairing rules. ${ }^{1}$ These advantages have been exploited in the field of nanotechnology to control nanoparticle properties by assembling them into supramolecular materials. ${ }^{2,3}$ The structure and properties of such materials are known to be sensitive to DNA interactions with counterions such as sodium or magnesium. Cations are commonly used to facilitate DNA hybridization by screening electrostatic repulsion between the DNA phosphate backbone, and are especially important for nanoparticle assemblies in which DNA strands are densely packed. ${ }^{4,5}$ Charge screening using counterions has been shown to enhance the properties of nanoparticle assemblies that derive from nanoparticle coupling, ${ }^{6}$ and in some cases give rise to novel properties of nanoparticle assemblies such as cooperative melting ${ }^{5,7}$ and resistance to nucleases. ${ }^{8}$ On the other hand, counterion sensitivity also limits downstream applications of DNA-assembled nanoparticles. First, it renders them structurally labile ${ }^{9,10}$ and incompatible with some solid-state fabrication processes. Second, while high ionic strength solvents have previously been used to enhance the properties of DNA- assembled nanoparticles such as plasmon coupling, ${ }^{3,6,11}$ high salts can also induce nanoparticle aggregation, making this strategy unsuitable for solution-based applications. Finally, for biological uses such as intracellular sensing and drug delivery, counterion concentration is dependent on the physiological environment and beyond user control.

Here we show that coating DNA-assembled nanoparticles with cationic polymers can enhance their structure and properties beyond the native environment from which they were synthesized. Specifically, we show that substituting sodium or magnesium cations with polyamines of specific lengths rigidified DNA-assembled nanoparticles and reduced interparticle spacing. In the case of metal nanoparticles, this reduction in interparticle spacing also enhanced plasmon coupling. Similar to how histones package DNA in nucleosomes, these novel properties result from the ability of polyvalent amines to condense DNA. We further show that polyamine coated DNA assemblies provide a scaffold to create new materials based on the layer-by-layer assembly method. We

Received: January 21, 2016

Published: March 4, 2016 
propose that in general, polyvalent counterions can be designed to engineer the structure and properties of DNA-assembled nanostructures for downstream applications.

\section{RESULTS AND DISCUSSION}

DNA Assembly of Metal Nanoparticle Superstructures. This study focused on a specific nanoparticle assembly geometry commonly referred to as "core-satellite" (Figure 1A). This geometry features a number of tunable parameters

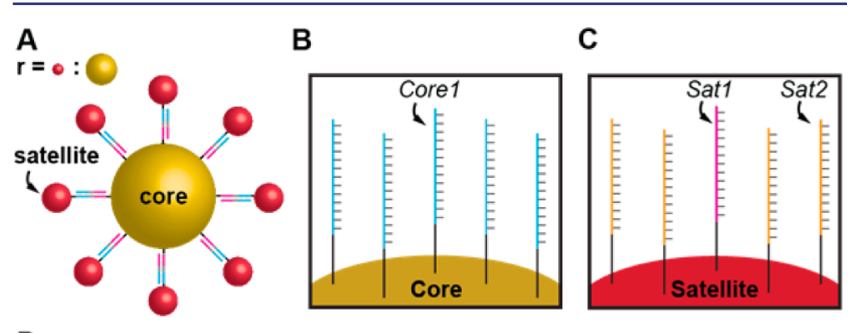

D

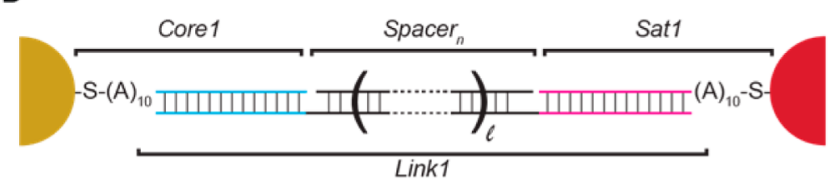

E

$\mathbf{F}$
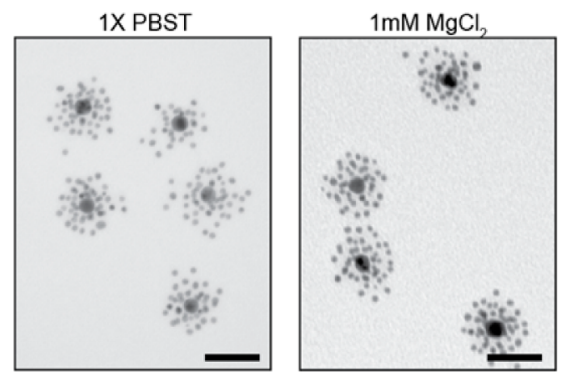

G

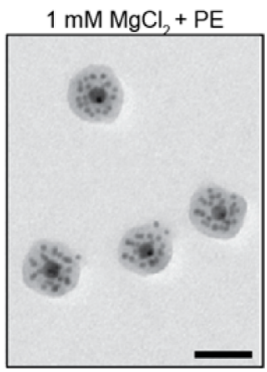

Figure 1. DNA assembly of gold nanoparticles into "core-satellite" superstructures. (A) Schematic of the superstructure showing satellite nanoparticles in red and core nanoparticle in yellow. Parameters varied in this study were diameters of the core and satellite $\left(d_{\text {core }}\right.$ and $\left.d_{\text {sat }}\right)$ as well as their stoichiometry $(r)$. (B) Core nanoparticles were grafted with a single $25 \mathrm{nt}$ sequence (Core1) while (C) satellites were grafted with two $25 \mathrm{nt}$ sequences (Sat1 and Sat2). (D) Link1 was used to connect Core 1 to Sat1 separated by $60 \mathrm{nt}$. This single-stranded region was used to attach a number of spacer strands (denoted Spacer $_{n}$ ) conjugated with fluorophores. (E) Representative TEMs of coresatellite superstructures after resuspension in $1 \times$ PBST, (F) $1 \mathrm{mM}$ $\mathrm{MgCl}_{2}$, and after $(\mathrm{G})$ polyelectrolyte coating $(\mathrm{PE})$ in $1 \mathrm{mM} \mathrm{MgCl}$. Scale bars $=50 \mathrm{~nm}$.

which have been theoretically studied in the context of plasmon engineering $^{12}$ as well as experimentally implemented using various types of linkers, ${ }^{12-16}$ making it a well-defined model system. As building blocks for assembling core-satellite superstructures, we synthesized gold nanorods and spherical gold nanoparticles of 5, 15,30, and $60 \mathrm{~nm}$ in diameter and grafted them with single-stranded, thiolated oligonucleotides according to published procedures. ${ }^{17-19}$ Nanoparticles designated as the "core" were grafted with a 25 nt oligonucleotide sequence (Core1) for linking to "satellite" nanoparticles (Figure 1B). Nanoparticles designated as "satellites" were grafted with two different sequences of oligonucleotides of $25 \mathrm{nt}$ each (Sat1 and Sat2, Figure 1C). In this mixed monolayer, Sat1 was used for the purpose of assembly while Sat2 is a noncomplementary sequence used to fill vacant binding sites on the satellite nanoparticle surface. We kept the Sat1-to-Sat2 grafting stoichiometry low (1:4) to minimize cross-linking superstructures into macroscopic aggregates. This mixed monolayer strategy was useful for maximizing the surface charge density of satellite nanoparticles, which improved their colloidal stability and subsequent interactions with cationic polyelectrolytes. Oligonucleotide grafting onto nanoparticles was performed according to the $\mathrm{pH}$-assisted method of Liu et al., ${ }^{19}$ which we found to be superior to the conventional salt-aging method in that similar grafting densities were obtained within $30 \mathrm{~min}$ of incubation instead of hours to days (e.g., $85 \pm 6.0$ strands per $15 \mathrm{~nm}$ nanoparticle and $66 \pm 2.4$ strands per nanorod, see Figure S1). To initiate assembly, "core" nanoparticles were first incubated with an excess of 90 nt linker strand (Link1, Figure 1D), isolated by centrifugation, and then incubated with $100 \times$ molar excess of "satellite" nanoparticles. Link1 contains a singlestranded region that binds one to several spacer strands (denoted Spacer $_{n}$ in Figure 1D), which we used to incorporate other molecules within the core-satellite. Assembled superstructures were washed by centrifugation and stored in phosphate buffered saline containing $0.01 \% \mathrm{w} / \mathrm{v}$ Tween 20 (PBST). Transmission electron microscopy (TEM) showed that superstructures were uniform in size and well dispersed (Figure 1E and Figure S2).

Polyamines Compact DNA-Metal Superstructures. To integrate core-satellite superstructures with polyamines, we first buffer exchanged them into $1 \mathrm{mM} \mathrm{MgCl}$. This step was important, since initial attempts at exposing superstructures to various types of cationic polyelectrolytes in PBST resulted in colloidal aggregation and precipitation (Figure S3A,B). In contrast, the presence of low millimolar concentrations of magnesium maintained both the stability of the DNA duplex and the colloidal stability of DNA-assembled metal nanoparticle superstructures as determined by fluorescence and spectrophotometric assays (Figure S3C,D), as well as TEM (Figure 1F). Buffer exchanged superstructures were added dropwise into a solution of cationic polyelectrolytes such as poly(allylamine) and vigorously stirred for 1 to $2 \mathrm{~h}$. Superstructures were purified from excess polyelectrolytes by centrifugation and reexamined under TEM (Figure 1G). Comparing superstructure morphology before and after polyelectrolyte coating, we noted two interesting observations. First, polyelectrolyte coating preserved the discrete, colloidal nature of the superstructures and the core-satellite morphology. Second, coating reduced the spacing between nanoparticles and the overall size of the superstructures. These observations were confirmed by dynamic light scattering measurements, which showed unimodal size distribution and a decrease in the hydrodynamic size of the superstructures following polyelectrolyte coating (Figure 2A). For core-satellites assembled using 15 and $5 \mathrm{~nm}$ gold nanoparticles, the hydrodynamic diameter decreased from 87 to $62 \mathrm{~nm}$ after polyelectrolyte coating. On the basis of a quasi-spherical geometry, this size reduction corresponded to a remarkable 2.8-fold volumetric compression, consistent with the known ability of polyvalent cations to condense DNA. ${ }^{20-23}$

To confirm the condensation of DNA in solution, we attached Cy5-labeled spacer strands (Spacer 2 -Cy5) to Core1/ Link1-functionalized $15 \mathrm{~nm}$ gold nanoparticles (Figure 2B). These particles were then incubated with poly(allylamine) as described in the previous section. Fluorescence measurements showed that polyelectrolyte coating decreased Cy5 fluorescence intensity by $87 \%$ compared to noncoated nanoparticles (Figure 2B). Since metals quench fluorophores in a distance-dependent 
A

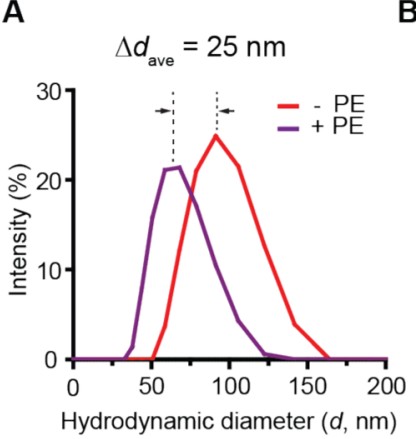

C

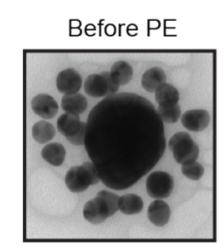

D

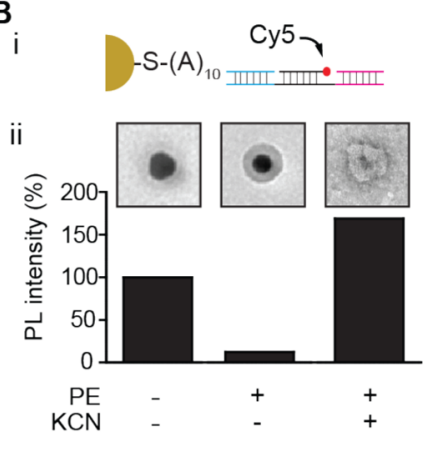

E

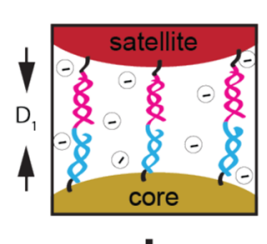

After PE
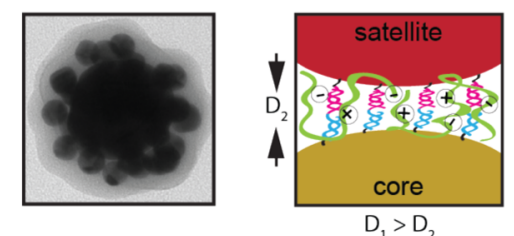

$D_{1}>D_{2}$

Figure 2. Compaction of polyelectrolyte coated core-satellites. (A) Hydrodynamic diameter of core-satellites $\left(d_{\text {core }}=15 \mathrm{~nm}, d_{\text {sat }}=5 \mathrm{~nm}\right.$, $r=30: 1$ ) before and after polyelectrolyte coating. (B) Fluorescence of Cy5-labeled oligonucleotides on the nanoparticle surface before and after polyelectrolyte and potassium cyanide treatment. (i) Position of the Cy5 label. (ii) Cy5 fluorescence and corresponding TEM of materials following treatment. (C) TEM of core-satellite $\left(d_{\text {core }}=60\right.$ $\left.\mathrm{nm}, d_{\text {sat }}=30 \mathrm{~nm}, r=30: 1\right)$ before and after polyelectrolyte coating. (D,E) Proposed model of two mechanisms by which polyelectrolytes interact with and compact the structure. PE: polyelectrolyte coat; KCN: potassium cyanide.

manner, ${ }^{24-26}$ this result suggests that Cy5-labeled oligonucleotides moved closer to the nanoparticle surface following polyelectrolyte coating. Removal of the gold core by potassium cyanide dissolution restored the fluorescence by $150 \%$, confirming that fluorophore interactions with the metal (e.g., not the amines) $)^{27}$ accounted for the majority of quenching. We further confirmed structural compaction as a result of DNA condensation using TEM. Figure $2 \mathrm{C}$ shows representative images of superstructures consisting of $60 \mathrm{~nm}$ core and $15 \mathrm{~nm}$ satellite nanoparticles, before and after exposure to poly(allylamine). The polymer layer appeared as a shell around the superstructure, and reduced spacing both between core and satellites as well as between neighboring satellite nanoparticles. On the basis of these observations, we propose that polyamines can (1) compact DNA nanostructures through DNA condensation (Figure 2D), and (2) cross-link neighboring nanoparticles through polyvalent interactions with multiple DNA strands (Figure 2E). The latter further contributes to structural compaction, and may also improve the mechanical stability of the nanostructure.

Polyelectrolytes Enhance Plasmon Coupling of DNAMetal Superstructures. We hypothesized that the ability of polyamines to compact metal superstructures may enhance plasmon-coupling efficiency, which is known to vary as a function of interparticle distance. To test this, we fabricated two sets of assembly designs each consisting of $60 \mathrm{~nm}$ gold nanoparticle cores linked with either 15 or $30 \mathrm{~nm}$ satellites (Figure $3 \mathrm{~A}, \mathrm{~B})$. The relatively large core size was chosen based on the motivation that its large extinction cross-section would allow us to directly visualize plasmon coupling enhancement, whereas the two different satellite sizes would allow us to compare the effects of assembly design on plasmon coupling efficiency following interactions with polyelectrolytes. Indeed, upon exposure to polyelectrolytes, we observed immediate change in the color of the colloidal solutions changed from red to different hues of blue and purple (Supplementary Video 1). The final colors varied as a function of both the size of the core and satellite nanoparticles and their assembly stoichiometry (Figure 3C,D). These differences were quantified using UV-vis spectrophotometry as shown in Figures 3E,F. Before polyelectrolyte addition, the absorption spectra of both sets of designs were dominated by a single peak at $550 \mathrm{~nm}$, associated with the surface plasmon resonance mode of single spherical $60 \mathrm{~nm}$ gold nanoparticles (Figure 3E,F, "before PE"). For both satellite designs, the addition of polyelectrolytes resulted in significant redshift in the surface plasmon resonance absorption, and in broadening of the absorption line width. These patterns are indicative of increased dampening of plasmon charge oscillations and the generation of higher order plasmon modes, reflecting enhanced plasmon coupling between assembled nanoparticles. Polyelectrolytes also augmented the impact of design on the optical response of the nanostructures. Figure $3 \mathrm{E}$ shows that increasing satellite-to-core stoichiometry from 3 to 30 in the absence of polyelectrolytes corresponded to a $5 \mathrm{~nm}$ redshift in UV-vis absorption. In contrast, the same design red-shifted by $70 \mathrm{~nm}$ following polyelectrolyte addition (Figure 3E, "after PE"). Similar trends could be observed for the $30 \mathrm{~nm}$ satellite design by comparing the "Before PE" panel to the "After PE" panel in Figure 3F. Overall, the largest enhancement was observed in assemblies consisting of $60 \mathrm{~nm}$ core and $30 \mathrm{~nm}$ satellite nanoparticles with the highest satellite-to-core stoichiometry. Here, the addition of polyelectrolytes generated a new absorption peak at $670 \mathrm{~nm}$, corresponding to a $120 \mathrm{~nm}$ redshift from the original peak of $550 \mathrm{~nm}$ (Figure 3F, v).

Interestingly, we reproduced these same trends using poly $(\mathrm{L}-$ lysine) as another cationic polyelectrolyte, suggesting that enhancement is not restricted to one type of polyamine. We further examined the effects of polyamine length on plasmon coupling by coating DNA-metal superstructures with poly(Llysine) consisting of 5, 10, 30, 100, or 250 repeat units (Figure S4). UV-vis spectrophotometry showed sharp transition in enhancement at a chain length of 10 monomers. Chain lengths longer than 10 monomers led to diminishing increases in plasmon enhancement, whereas chain length smaller than 10 did not result in enhancement. These observations are consistent with the hypothesis that the degree of DNA condensation controls structural compaction and plasmon enhancement, and that these properties are tunable as a function of polyamine length (e.g., valency).

Layer-by-Layer Encapsulation of DNA-Metal Superstructures. Polyamine coated DNA-metal superstructures exhibited uniform morphology and excellent colloidal stability. This prompted us to hypothesize that in general, DNA nanostructures can be used as supramolecular scaffolds to template polyelectrolyte deposition using the layer-by-layer 
A

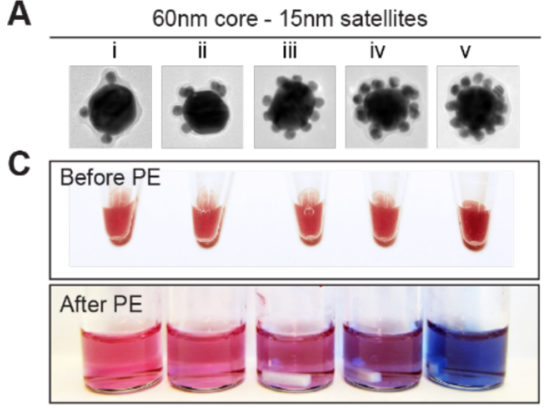

E
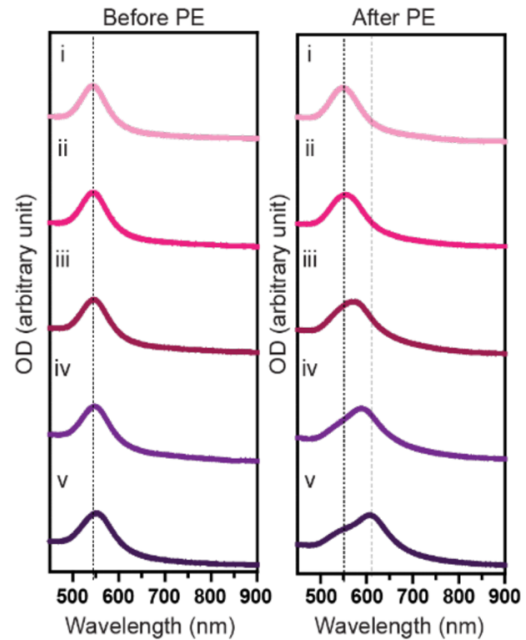

B

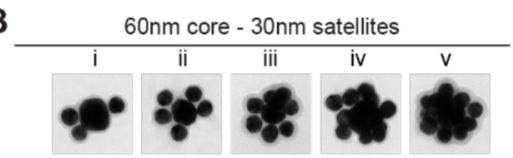

D
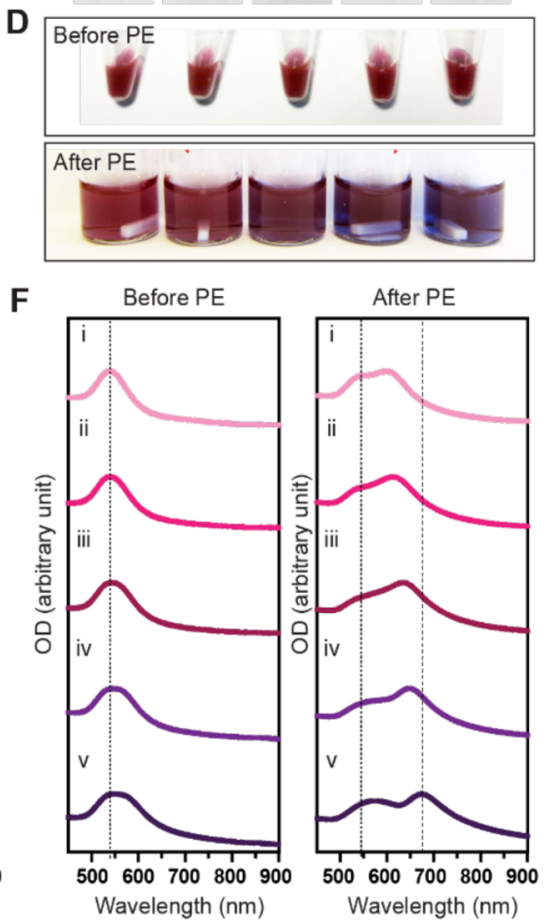

Figure 3. Polyelectrolyte coating enhances plasmon coupling of core-satellites. (A,B) TEM of two sizes of core-satellite structures, with satellite-tocore stoichiometry increasing from 3:1 to 30:1 (i-v). (C,D) Corresponding colors of the core-satellite solutions before and after polyelectrolyte coating (PE). (E,F) Corresponding UV-vis absorbance spectra of the core-satellite solutions shown in C and D. OD: optical density.

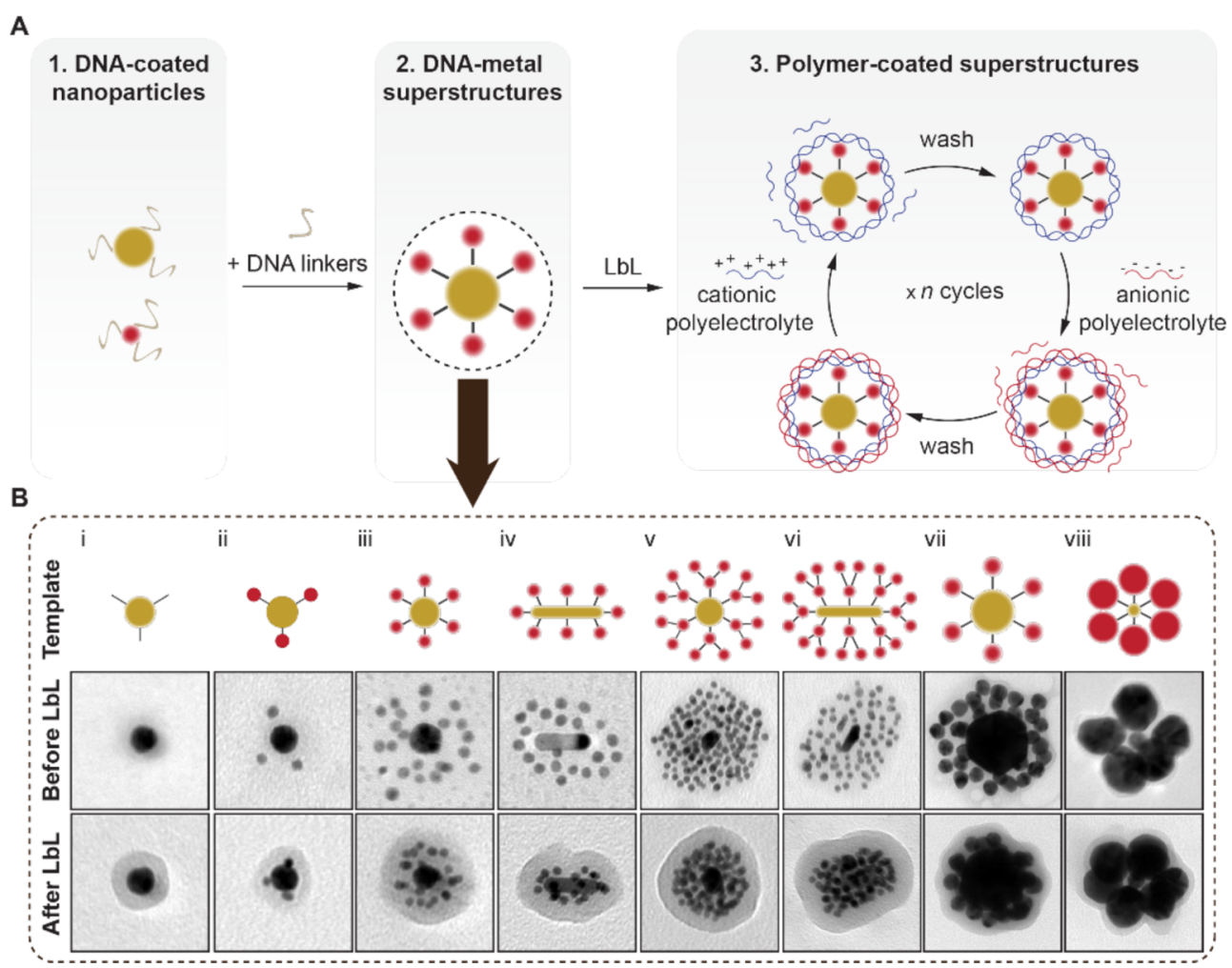

Figure 4. Encapsulation of metal superstructures using the layer-by-layer technique. (A) Schematic of the layer-by-layer fabrication procedure. (B) Morphological transition of eight different metal superstructures before and after coating with four cycles of poly(allylamine) and poly(styrenesulfonate). LbL: layer-by-layer. 
assembly method ${ }^{28,29}$ (Figure 4A). If so, polyelectrolyte engineering and deposition would be a versatile synthetic strategy to improve the structural integrity and functionality of DNA-based nanomaterials. To test this, we performed layer-bylayer assembly on a panel of eight DNA-assembled metal nanostructures with varying size, shape, and assembly architectures, using poly(allylamine) and poly(styrenesulfonate) as the model polyelectrolyte pair (Figure 4B). Under TEM, we observed successful growth of polyelectrolyte multilayers around all the structures tested, evidenced by the appearance of a uniform polymer shell (Figure 4B, “after LbL"). Zeta potential measurements further demonstrated robust surface charge reversal following each round of deposition (Figure S5). Similar to our previous results, polyelectrolyte deposition induced structural compaction in all the DNA-metal superstructures while preserving the overall geometry of the assembly (Figure 4B).

We further characterized three selected layer-by-layer coated nanostructures by scanning electron microscopy (Figure 5A, i-

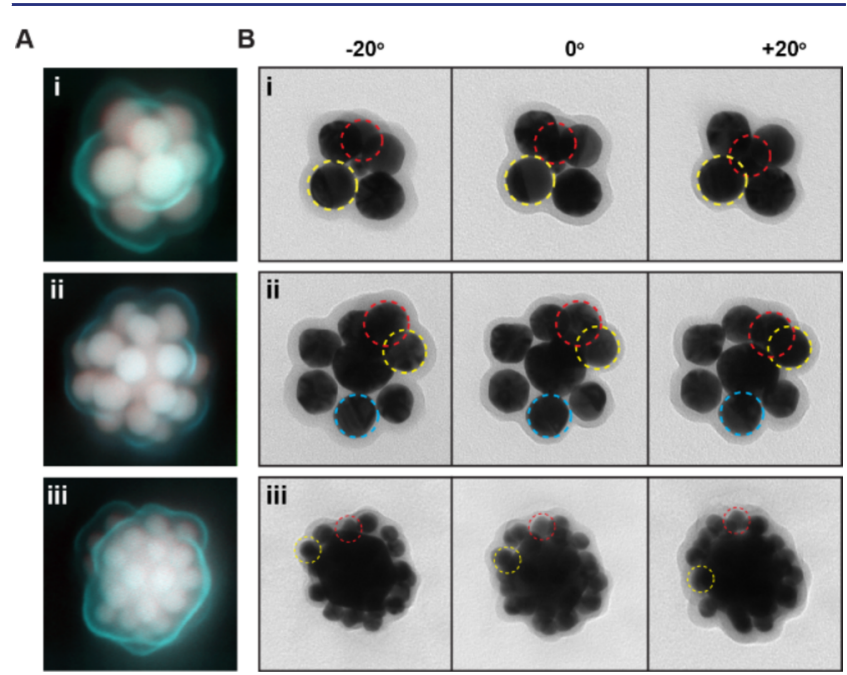

Figure 5. Structural examination of layer-by-layer coated DNA-metal superstructures. (A) SEM of three different superstructures following LbL assembly: (i) $d_{\text {core }}=15 \mathrm{~nm}, d_{\text {sat }}=30 \mathrm{~nm}, r=6: 1$; (ii) $d_{\text {core }}=60$ $\mathrm{nm}, d_{\mathrm{sat}}=30 \mathrm{~nm}, r=20: 1$; (iii) $d_{\text {core }}=60 \mathrm{~nm}, d_{\text {sat }}=15 \mathrm{~nm}, r=30: 1$. Polyelectrolyte coatings are false-colored in cyan and nanoparticles in white. (B) Tilt-angle TEM showing the 3D architecture of the superstructures following polyelectrolyte coating. (i-iii) correspond to the three designs shown in (A).

iii). By separately detecting both secondary and backscattered electrons, the polyelectrolyte coating (cyan) could be clearly distinguished from the inorganic nanoparticles (white). Although each superstructure displayed a quasi-spherical morphology overall, the polyelectrolyte can be seen to wrap around the contour of individual nanoparticles, suggesting they cross-link nanoparticles through electrostatic interactions with the attached DNA. To better understand the morphology of layer-by-layer coated nanostructures, we characterized them by tilted-angle TEM, in which the plane of observation was rotated about the $z$-axis by a total of $40^{\circ}$ (Figure 5B, i-iii). Comparing change in nanoparticle orientation as a function of tilt angle (e.g., dotted circles in Figure 5B) with nonpolyelectrolyte coated controls, we believe that polyelectrolyte multilayers also enhanced the structural rigidity of DNA-metal superstructures (see discussion in Figure S6).
Polyelectrolyte Composition Adds Functionality to DNA-Metal Superstructures. Plasmonic nanostructures have applications as biological imaging agents and sensors, but nanostructures assembled from DNA lack long-term stability and are destroyed quickly by endogenous nucleases. In contrast, we found that layer-by-layer coated DNA-metal nanoparticle superstructures remained stable for at least one month when stored in $1 \mathrm{mM} \mathrm{MgCl}$ or deionized water at $4{ }^{\circ} \mathrm{C}$, as determined by TEM (Figure S7). Coating also protected superstructures against nuclease degradation as demonstrated by a DNase I assay (Figure S8). Enzymatic degradation was tunable as a function of polyelectrolyte composition. For example, trypsin is a serine protease that breaks down proteins by catalyzing the hydrolysis of peptide bonds. For superstructures coated with the polypeptides poly(L-lysine) and poly(L-glutamic acid), incubation with trypsin resulted in blueshift of their plasmon absorption pattern back to the state before layer-by-layer assembly. This was accompanied by disappearance of the polyelectrolyte layer around the superstructures when examined under TEM (Figure 6B, from state i

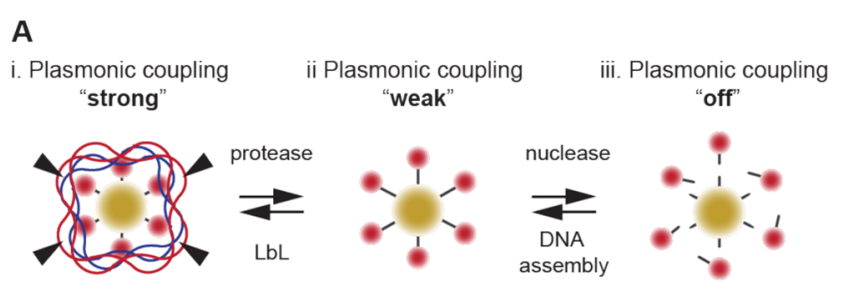

B

$60 \mathrm{~nm}$ core $-30 \mathrm{~nm}$ satellites

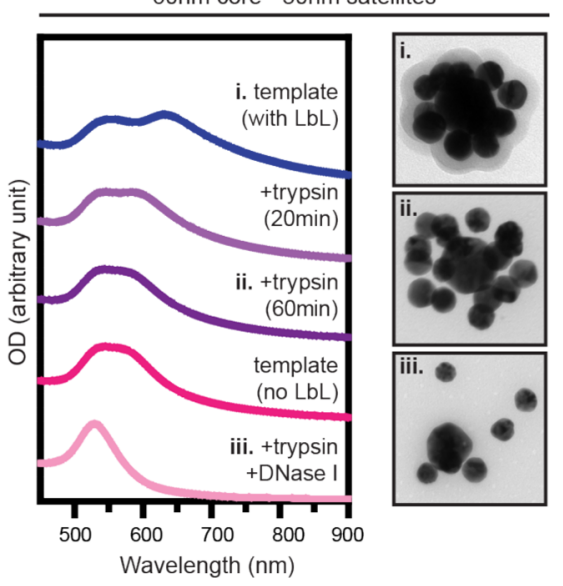

Figure 6. Tuning plasmonic response of LbL-encapsulated metal superstructures to environmental stimuli. (A) Three distinct plasmoncoupling states in response to protease and nuclease degradation. (B) UV-vis absorbance spectra and corresponding TEM of core-satellites $\left(d_{\text {core }}=60 \mathrm{~nm}, d_{\text {sat }}=30 \mathrm{~nm}, r=20: 1\right)$. Spectrum of the core-satellite without coating (no LbL) is included for reference. LbL: layer-bylayer.

to state ii). Further blueshift in the absorption spectra to that of the nonassembled state, was observed with the addition of both DNase I and trypsin (Figure 6B, from state ii to iii), suggesting that enzyme combinations can toggle different optical responses. On the other hand, for superstructures coated with poly(allylamine) and poly(styrene-sulfonate), which do not contain peptide bonds in their backbone, the optical response of the superstructures was unchanged after incubation with either DNase I or trypsin, or a combination of these enzymes (Figure S9). Together, these results highlight the use of 
polyelectrolyte composition to customize the structure and properties of DNA-assembled nanoparticles for downstream applications.

\section{CONCLUSION}

In this study, we showed that polyamines enhance the structural rigidity and plasmon coupling of DNA-metal superstructures through nanoparticle cross-linking and DNA condensation. Unlike sodium and magnesium ions, interaction between polyamines and DNA is polyvalent and long-lived, where the degree of avidity can be tuned as a function of polymer chain length. In this regard, the use of polyaminebased counterions for engineering the properties of DNAassemblies deserves further development. For example, future work can elucidate whether regimes of structural rigidity and compaction can be separated with the appropriate structure of polyamine, as the two effects can be desirable for different applications. The incorporation of other types of environmentally responsive polyamines and polyelectrolytes to DNAbased nanomaterials can also be useful. For example, thiol- or acid-responsive polyelectrolytes can be used to report on the intracellular location of plasmonic probes or the release of payloads through a change in the optical response. To engineer more complex optical properties, methods such as DNA origami could be employed to further control the geometry and composition of nanoparticle assembly. ${ }^{30}$ Integration of such nanostructures with polyamines merits further exploration. In conclusion, the appropriate design of counterion chemistry and fine-tuning of DNA-counterion interactions have important implications for practical applications of DNA nanostructures.

\section{EXPERIMENTAL PROCEDURES}

Materials. Sources of all the reagents used in this study are listed in Supporting Table S1.

Gold Nanoparticle Synthesis. Gold nanoparticles of $15 \mathrm{~nm}$ in diameter were synthesized by adding $\mathrm{HAuCl}_{4}(1 \mathrm{~mL}, 1 \% \mathrm{w} / \mathrm{v})$ to Nanopure water $(98 \mathrm{~mL})$ in a $250 \mathrm{~mL}$ Erlenmeyer flask. The solution was brought to a boil on a hot place set to $300{ }^{\circ} \mathrm{C}$, and trisodium citrate $(1 \mathrm{~mL}, 3 \% \mathrm{w} / \mathrm{v})$ was injected swiftly into the flask while the reaction mixture is under vigorous stirring. The reaction was allowed to take place for $7 \mathrm{~min}$ and then quenched on ice. Nanoparticles were washed by centrifugation at $4{ }^{\circ} \mathrm{C}$ and $13000 \mathrm{~g}$ for $30 \mathrm{~min}$ in Tween 20 $(0.01 \% \mathrm{w} / \mathrm{v})$.

Gold nanoparticles of $5 \mathrm{~nm}$ in diameter were synthesized by adding $\mathrm{HAuCl}_{4}(1 \mathrm{~mL}, 1 \% \mathrm{w} / \mathrm{v})$ to Nanopure water $(79 \mathrm{~mL})$ in a $250 \mathrm{~mL}$ Erlenmeyer flask heated on a hot plate at $60{ }^{\circ} \mathrm{C}$. A reducing solution was prepared by mixing water $(16 \mathrm{~mL})$, tannic acid $(1 \mathrm{~mL}, 1 \% \mathrm{w} / \mathrm{v})$, trisodium citrate $(4 \mathrm{~mL})$ and potassium bicarbonate $(1 \mathrm{~mL}, 3.46 \mathrm{mg} /$ $\mathrm{mL}$ ). The reducing solution was heated to $60{ }^{\circ} \mathrm{C}$ and injected swiftly into the reaction mixture under vigorous stirring. The reaction was allowed to take place at $60^{\circ} \mathrm{C}$ for $30 \mathrm{~min}$ and at $90{ }^{\circ} \mathrm{C}$ for $10 \mathrm{~min}$, and then quenched on ice. Nanoparticles were stabilized by overnight incubation with bis( $p$-sulfonatophenyl)-phenylphosphine dihydrate dipotassium salt $(0.8 \mathrm{mg} / \mathrm{mL})$ while stirring, and washed by ultracentrifugation two times at $4{ }^{\circ} \mathrm{C}$ and $250000 \mathrm{~g}$ for $30 \mathrm{~min}$ in Tween $20(0.01 \% \mathrm{w} / \mathrm{v})$.

Gold nanoparticles of $30 \mathrm{~nm}$ and $60 \mathrm{~nm}$ in diameter were synthesized according to previously published procedures. ${ }^{18}$ Briefly, 15 $\mathrm{nm}$ nanoparticle "seeds" were added to Nanopure $\mathrm{H}_{2} \mathrm{O}$ in a $250 \mathrm{~mL}$ Erlenmeyer flask, followed by addition of $\mathrm{HAuCl}_{4}(1 \mathrm{wt} \%)$ and trisodium citrate $(4.1 \mathrm{mg} / \mathrm{mL})$ as stabilizers. A solution of hydroquinone $(3.3 \mathrm{mg} / \mathrm{mL})$ was then swiftly injected into the solution under vigorous stirring to reduce gold ions onto seed surface. The reaction was allowed to take place overnight at room temperature for completion. Synthesized particles were washed three times with washing buffer containing sodium citrate $(0.1 \mathrm{mg} / \mathrm{mL})$ and Tween 20
$(0.05 \% \mathrm{w} / \mathrm{v})$. The size of the particle was controlled by changing the stoichiometry of seeds to gold solution as detailed in Supporting Table S2. Nanoparticles were washed three times by centrifugation at $4{ }^{\circ} \mathrm{C}$, using $4000 \mathrm{~g}$ for $30 \mathrm{~nm}$ and $1000 \mathrm{~g}$ for $60 \mathrm{~nm}$ nanoparticles for $30 \mathrm{~min}$ in Tween $20(0.01 \% \mathrm{w} / \mathrm{v})$.

Gold nanorods with aspect ratio of $4.1 \pm 0.2$ were synthesized from a seed solution created by adding $1.2 \mathrm{~mL}$ of sodium borohydride $(0.01$ M) to a $20 \mathrm{~mL}$ scintillation vial containing $500 \mu \mathrm{L}$ of $\mathrm{HAuCl}_{4}(0.01$ $\mathrm{M})$ and $19.5 \mathrm{~mL}$ of cetyltrimethylammonium bromide (CTAB, $0.1 \mathrm{M})$ under vigorous stirring. In a clean bottle, $49.5 \mathrm{~mL}$ of $0.01 \mathrm{M} \mathrm{HAuCl}_{4}$ was added to $950 \mathrm{~mL}$ of CTAB $(0.1 \mathrm{M})$. To this solution, $5 \mathrm{~mL}$ of $\mathrm{AgNO}_{3}(0.01 \mathrm{M})$ and $7 \mathrm{~mL}$ of ascorbic acid $(0.1 \mathrm{M})$ were added respectively under stirring. Finally, $20 \mathrm{~mL}$ of the above prepared seed solution was added and the entire solution was left overnight under stirring. The obtained gold nanorod solution was then purified by centrifugation twice at $17000 \mathrm{~g}$ for $20 \mathrm{~min}$ and redispersed in water to remove excess CTAB.

Synthesized nanoparticles were characterized using TEM, UV-vis spectrophotometry, and dynamic light scattering.

Oligonucleotide Grafting. Spherical gold nanoparticles were functionalized with thiolated DNA using a previously published method. ${ }^{19}$ Briefly, $15 \mathrm{~nm}$ gold nanoparticles $(25 \mathrm{nM})$ were mixed with thiolated Core1 $(3.75 \mu \mathrm{M})$ in the presence of Tween $20(0.01 \% \mathrm{w} / \mathrm{v})$ and trisodium citrate $(30 \mathrm{mM}, \mathrm{pH} 3.0)$. The mixture was incubated for $30 \mathrm{~min}$ and DNA functionalized gold nanoparticles were purified by centrifugation at $16000 \mathrm{~g}$ for $30 \mathrm{~min}$ at $4{ }^{\circ} \mathrm{C}$ using $1 \times$ phosphate buffered saline containing Tween $20(0.01 \% \mathrm{w} / \mathrm{v})$ as the solvent. Five $\mathrm{nm}$ gold nanoparticles $(100 \mathrm{nM})$ were coated with a mixture of thiolated Sat1 and Sat2 (ratio $=3: 7,2 \mu \mathrm{M}$ overall) using the same buffer as for the $15 \mathrm{~nm}$ gold nanoparticles. Oligonucleotide coated 5 $\mathrm{nm}$ nanoparticles were washed and isolated by ultracentrifugation two times at $250000 \mathrm{~g}$ for $30 \mathrm{~min}$ at $4{ }^{\circ} \mathrm{C}$ in $1 \times$ PBST. Samples were stored at $4{ }^{\circ} \mathrm{C}$. The amount of oligonucleotides used for grafting larger spherical nanoparticles was scaled to maintain constant oligonucleotide concentration per nanoparticle surface area.

Gold nanorods were washed twice in water by centrifugation at $13000 \mathrm{~g}$ for $30 \mathrm{~min}$ at room temperature. Nanorods were next added to solution containing sodium dodecyl sulfate $(0.2 \% \mathrm{w} / \mathrm{v})$ and thiolated oligonucleotides in $500 \times$ molar excess. A solution of $2 \times$ trisborate-EDTA (TBE) containing sodium chloride $(1 \mathrm{M})$ at $\mathrm{pH} 3.0$ was added in equal volume to the nanorod solution to facilitate the grafting of oligonucleotides onto the nanorod. The solution was incubated overnight in a $37{ }^{\circ} \mathrm{C}$ bath, and then washed twice at $15000 \mathrm{~g}$ for 35 $\min$ at $30{ }^{\circ} \mathrm{C}$ using $1 \times$ PBS with $0.01 \% \mathrm{w} / \mathrm{v}$ Tween 20 . The concentration of the nanorods was measured by UV-vis absorbance at $740 \mathrm{~nm}$. Samples were stored at $4{ }^{\circ} \mathrm{C}$ prior to use.

Nanoparticle Grafting Density. Density of oligonucleotides on nanoparticles was determined as previously described. ${ }^{31}$ Briefly, nanoparticle concentration was determined by UV-vis spectrophotometry using published molar extinction coefficients of the nanoparticles. ${ }^{32}$ Nanoparticles were then incubated with dithiothreitol (10 $\mathrm{mM}$ ) at $60{ }^{\circ} \mathrm{C}$ for $30 \mathrm{~min}$ to liberate the grafted oligonucleotides and pelleted by centrifugation at $16000 \mathrm{~g}$ for $30 \mathrm{~min}$ to remove the aggregates. Oligonucleotide concentration in the supernatant was determined using the Oligreen reagent and compared with standards that have undergone identical treatment. The average oligonucleotides grafting density per nanoparticle was determined by dividing the oligonucleotide concentration to the nanoparticle concentration.

Nanoparticle Assembly. DNA assembly was performed according to previously published methods with some modifications. ${ }^{31}$ Briefly, Core 1 oligonucleotide functionalized $15 \mathrm{~nm}$ gold nanoparticles (10 $\mathrm{nM})$ were mixed with Link1 oligonucleotides $(1 \mu \mathrm{M})$ in hybridization buffer consisting of $3 \times$ phosphate buffered saline containing magnesium chloride $(5 \mathrm{mM})$ and Tween $20(0.01 \% \mathrm{w} / \mathrm{v})$. The mixture was incubated in a $60^{\circ} \mathrm{C}$ water bath for $10 \mathrm{~min}$ and then incubated in a $37^{\circ} \mathrm{C}$ incubator for $1 \mathrm{~h}$. The mixture was then taken out and cooled to room temperature for $20 \mathrm{~min}$, and washed by centrifugation 2 times at $4{ }^{\circ} \mathrm{C}$ and $16000 \mathrm{~g}$ for $30 \mathrm{~min}$ using $1 \times$ PBST to remove excess Link1 oligonucleotides. 
Purified Link1-functionalized nanoparticles $(10 \mathrm{nM})$ were mixed with spacer strands Spacer1 and Spacer2 or their fluorophore-labeled versions at 100:1 molar excess relative to nanoparticles in hybridization buffer. Particles were incubated in a $37^{\circ} \mathrm{C}$ incubator for $1 \mathrm{~h}$. Satellite nanoparticles suspended in hybridization buffer were then added to this mixture at 100:1 molar excess relative to the core nanoparticles, and incubated at $37{ }^{\circ} \mathrm{C}$ for $1 \mathrm{~h}$. Assembled superstructures were cooled at room temperature for $30 \mathrm{~min}$, and then washed 4 times by centrifugation at $11000 \mathrm{~g}$ and $4{ }^{\circ} \mathrm{C}$ for $30 \mathrm{~min}$ using $1 \times$ PBST. Finally, superstructures $(2 \mathrm{nM})$ were incubated with Link2 $(0.4 \mu \mathrm{M})$ in hybridization buffer at $37^{\circ} \mathrm{C}$ for $1 \mathrm{~h}$, followed by washing three times in $1 \times$ PBST at $4{ }^{\circ} \mathrm{C}$ and $16000 \mathrm{~g}$ for $30 \mathrm{~min}$. Link2 was used to improve colloidal stability of the assembly in solution. After the last wash, superstructures were stored at $4{ }^{\circ} \mathrm{C}$ prior to use. The assembly of larger sizes of spherical nanoparticles followed the same procedures, with the single modification that nanoparticle concentration was reduced to maintain constant total surface area. For example, we used approximately $16 \times$ lower concentration of $60 \mathrm{~nm}$ core gold nanoparticles $(0.6 \mathrm{nM})$ in the assembly reaction than $15 \mathrm{~nm}$ core gold nanoparticles $(10 \mathrm{nM})$.

Assay of Colloidal Stability for Polyelectrolyte Coating. DNA-functionalized gold nanoparticles were suspended in water and mixed with aqueous solutions of cationic polyelectrolytes $(8 \mathrm{mg} / \mathrm{mL})$ titrated with different concentrations of either sodium chloride or magnesium chloride. Three compositions of polyelectrolytes, namely poly(L-lysine), poly(L-arginine), and poly(allylamine), were tested in parallel. The mixtures were incubated at room temperature for $1 \mathrm{~h}$. An aliquot from each mixture was centrifuged at $13000 \mathrm{~g}$ for $30 \mathrm{~min}$ to pellet the nanoparticles and resuspended in the same solvent. Colloidal stability was evaluated by measuring the UV-vis absorbance profile of the nanoparticle solutions before and after centrifugation. The extent of aggregation was determined by taking the ratio of absorbance at 520 to $700 \mathrm{~nm}$. Results were compared to controls of nanoparticles in water.

Assay of dsDNA Stability on Nanoparticles. FAM-labeled Linkl was attached to Core1-functionalized gold nanoparticles as described in the previous section. 8-methoxypsoralen $(20 \mathrm{mM}$ in DMSO) was added to nanoparticles to a final concentration of 500 $\mu \mathrm{M}$. Nanoparticles were exposed to UV on ice using a hand-held UVlamp for 30 to $120 \mathrm{~min}$ to induce UV cross-linking of oligonucleotides. Following cross-linking, nanoparticles were buffer exchanged into either water, $1 \mathrm{mM}$ magnesium chloride, or sodium chloride by centrifugation two times. Oligonucleotides that remained on the nanoparticle were removed by addition of freshly prepared dithiothreitol $(100 \mathrm{mM})$ followed by incubating the mixture at 60 ${ }^{\circ} \mathrm{C}$ for $30 \mathrm{~min}$. Nanoparticle aggregates were pelleted. FAM-labeled oligonucleotide standards were prepared using identical procedures and used for quantification.

Polyelectrolyte Coating. Before polyelectrolyte coating, DNAassembled metal nanoparticle superstructures were buffer exchanged into $1 \mathrm{mM}$ magnesium chloride by centrifugation. The buffered exchanged nanoparticle solution $(0.1 \mathrm{~mL}, 10 \mathrm{nM})$ was then dropwise added to $1 \mathrm{mM}$ magnesium chloride solution containing polyelectrolytes $(1 \mathrm{~mL}, 8 \mathrm{mg} / \mathrm{mL})$ under vigorous stirring. For a single layer coating, either poly(L-lysine) or poly(allylamine) was used as the cationic polyelectrolyte to complex with the anionic oligonucleotides displayed on the surface of the nanoparticles. The solution was stirred for $1 \mathrm{~h}$ before washing twice by centrifugation at $8000 \mathrm{~g}$ and $4{ }^{\circ} \mathrm{C}$ for 45 min using $1 \mathrm{mM}$ magnesium chloride as solvent. For layer-by-layer assembly, the aforementioned polyelectrolyte coating procedure was repeated using polyelectrolytes of alternating charges.

Experiments with different molecular weights of poly(L-lysine) were performed similarly with slight modifications. Poly(L-lysine) with chain length $(n)$ of $5,10,30,100$, or 250 were dissolved in water, $\mathrm{pH}$ adjusted to 7 , and $5 \times$ serially diluted from $10 \mathrm{mg} / \mathrm{mL}$ to $0.001 \mathrm{mg} /$ $\mathrm{mL}$. Nanoparticles were added to these working solutions the same way as described above and subsequently purified by centrifugation.

Scanning Electron Microscopy. Scanning electron micrographs of nanoparticle assemblies shown in Figure $5 \mathrm{~A}$ were obtained using a Hitachi S-5200 high resolution SEM equipped with two detectors to detect both secondary (SE) and backscattered electrons (BSE). SE mode was used to image the organic polyelectrolyte coating, while the gold nanoparticles were imaged under BSE mode. Images were false colored separately in ImageJ (nanoparticles in white, polyelectrolyte coating in cyan) and combined to visualize the coating.

Trypsin and DNase I Treatment. Superstructures $(10 \mu \mathrm{L}, 0.1$ $\mathrm{nM})$ were incubated either with trypsin $(10 \mu \mathrm{L}, 0.1 \% \mathrm{v} / \mathrm{v})$, DNase I $(10 \mu \mathrm{L}, 1 \mathrm{U} / \mu \mathrm{L})$ or both in $1 \times \mathrm{PBST}$ at $37{ }^{\circ} \mathrm{C}$ for $60 \mathrm{~min}$. The stability of the polyelectrolyte shell and the integrity of the DNA assembly were evaluated by monitored by measuring the UV-vis absorbance of the material. Samples treated with trypsin, DNase I or both were directly drop casted onto copper grids for TEM.

\section{ASSOCIATED CONTENT}

\section{Supporting Information}

The Supporting Information is available free of charge on the ACS Publications website at DOI: 10.1021/jacs.6b00751.

Supporting Figures S1-9, synthesis recipes for 30 and 60 $\mathrm{nm}$ gold nanoparticles, and list of materials and oligonucleotides used in this study. (PDF)

Supporting movie. (MPG)

\section{AUTHOR INFORMATION}

\section{Corresponding Author}

*warren.chan@utoronto.ca

\section{Present Address}

"Wyss Institute for Biologically Inspired Engineering, Harvard University, CLSB 5th floor, Three Blackfan Circle, Boston, Massachusetts 02115, United States.

Author Contributions

${ }^{\nabla}$ L.Y.T.C and F.S. contributed equally.

Notes

The authors declare no competing financial interest.

\section{ACKNOWLEDGMENTS}

The authors would like to acknowledge the Canadian Institute of Health Research (MOP-130143; RMF-111623; GCS 105653-1), Natural Sciences and Engineering Research Council (2015-06397), and Prostate Cancer Canada (D2014-12) for research support. L.C. would like to acknowledge the Canadian Breast Cancer Foundation and NSERC for fellowship support. We would like to like to acknowledge Vahid Raeesi and Dr. Kyryl Zagorovsky for technical and experimental discussions.

\section{REFERENCES}

(1) Jones, M. R.; Seeman, N. C.; Mirkin, C. A. Science 2015, 347 (6224), 1260901.

(2) Tan, S. J.; Campolongo, M. J.; Luo, D.; Cheng, W. Nat. Nanotechnol. 2011, 6 (5), 268.

(3) Barrow, S. J.; Funston, A. M.; Wei, X.; Mulvaney, P. Nano Today 2013, $8(2), 138$

(4) Castro, C. E.; Kilchherr, F.; Kim, D.-N.; Shiao, E. L.; Wauer, T.; Wortmann, P.; Bathe, M.; Dietz, H. Nat. Methods 2011, 8 (3), 221.

(5) Randeria, P. S.; Jones, M. R.; Kohlstedt, K. L.; Banga, R. J.; Olvera de la Cruz, M.; Schatz, G. C.; Mirkin, C. A. J. Am. Chem. Soc. 2015, 137 (10), 3486.

(6) Lermusiaux, L.; Maillard, V.; Bidault, S. ACS Nano 2015, 9 (1), 978.

(7) Jin, R.; Wu, G.; Li, Z.; Mirkin, C. A.; Schatz, G. C. J. Am. Chem. Soc. 2003, 125 (6), 1643.

(8) Seferos, D. S.; Prigodich, A. E.; Giljohann, D. A.; Patel, P. C.; Mirkin, C. A. Nano Lett. 2009, 9 (1), 308.

(9) Park, S.-J.; Lazarides, A. A.; Storhoff, J. J.; Pesce, L.; Mirkin, C. A. J. Phys. Chem. B 2004, 108 (33), 12375. 
(10) Busson, M. P.; Rolly, B.; Stout, B.; Bonod, N.; Larquet, E.; Polman, A.; Bidault, S. Nano Lett. 2011, 11 (11), 5060.

(11) Barrow, S. J.; Funston, A. M.; Gómez, D. E.; Davis, T. J.; Mulvaney, P. Nano Lett. 2011, 11 (10), 4180.

(12) Ross, B. M.; Waldeisen, J. R.; Wang, T.; Lee, L. P. Appl. Phys. Lett. 2009, 95 (19), 193112.

(13) Dey, P.; Zhu, S.; Thurecht, K. J.; Fredericks, P. M.; Blakey, I. J. Mater. Chem. B 2014, 2 (19), 2827.

(14) Gandra, N.; Abbas, A.; Tian, L.; Singamaneni, S. Nano Lett. 2012, 12 (5), 2645.

(15) Gandra, N.; Singamaneni, S. Chem. Commun. 2012, 48 (94), 11540.

(16) Sebba, D. S.; Lazarides, A. A. J. Phys. Chem. C 2008, 112 (47), 18331.

(17) Perrault, S. D.; Chan, W. C. W. J. Am. Chem. Soc. 2009, 131 (47), 17042.

(18) Chou, L. Y. T.; Chan, W. C. W. Adv. Healthcare Mater. 2012, 1 (6), 714.

(19) Zhang, X.; Servos, M. R.; Liu, J. J. Am. Chem. Soc. 2012, 134

(17), 7266.

(20) Bloomfield, V. A. Curr. Opin. Struct. Biol. 1996, 6 (3), 334.

(21) Koltover, I.; Wagner, K.; Safinya, C. R. Proc. Natl. Acad. Sci. U. S. A. 2000, 97 (26), 14046.

(22) Widom, J.; Baldwin, R. L. J. Mol. Biol. 1980, 144 (4), 431.

(23) Vijayanathan, V.; Thomas, T.; Shirahata, A.; Thomas, T. J. Biochemistry 2001, 40 (45), 13644.

(24) Anger, P.; Bharadwaj, P.; Novotny, L. Phys. Rev. Lett. 2006, 96 (11), 113002

(25) Schneider, G.; Decher, G.; Nerambourg, N.; Praho, R.; Werts, M. H. V.; Blanchard-Desce, M. Nano Lett. 2006, 6 (3), 530.

(26) Dulkeith, E.; Morteani, A. C.; Niedereichholz, T.; Klar, T. A.; Feldmann, J.; Levi, S. A.; van Veggel, F. C. J. M.; Reinhoudt, D. N.; Möller, M.; Gittins, D. I. Phys. Rev. Lett. 2002, 89 (20), 203002.

(27) Van, S.-P.; Hammond, G. S. J. Am. Chem. Soc. 1978, 100 (12), 3895 .

(28) Li, Y.; Wang, X.; Sun, J. Chem. Soc. Rev. 2012, 41 (18), 5998.

(29) Tong, W.; Song, X.; Gao, C. Chem. Soc. Rev. 2012, 41 (18), 6103.

(30) Tian, Y.; Wang, T.; Liu, W.; Xin, H. L.; Li, H.; Ke, Y.; Shih, W. M.; Gang, O. Nat. Nanotechnol. 2015, 10 (7), 637.

(31) Chou, L. Y. T.; Zagorovsky, K.; Chan, W. C. W. Nat. Nanotechnol. 2014, 9 (2), 148.

(32) Jain, P. K.; Lee, K. S.; El-Sayed, I. H.; El-Sayed, M. A. J. Phys. Chem. B 2006, 110 (14), 7238. 